\title{
Screening for antibodies against intact cancer cells with a naïve large phage antibody library
}

\author{
YUANYUAN QIAO ${ }^{1,2^{*}}$, LIJUN ZHOU ${ }^{2 *}$, YUXIAO WANG $^{2}$, FANG LIU $^{3}$, PENG XIE $^{2}$, \\ YUPING CHEN $^{2}$, DAJIN ZHANG ${ }^{2}$ and XIAOHANG ZHAO ${ }^{2,3}$ \\ ${ }^{1}$ Graduate School of Southern Medical University, Guangzhou 510515; ${ }^{2}$ Center for Basic Medical Sciences, Navy General \\ Hospital of Chinese PLA, Beijing 100048; ${ }^{3}$ State Key Laboratory of Molecular Oncology, Cancer Institute and Hospital, \\ Chinese Academy of Medical Sciences and Peking Union Medical College, Beijing 100021, P.R. China
}

Received June 30, 2011; Accepted August 12, 2011

DOI: $10.3892 / \mathrm{ijmm} .2011 .800$

\begin{abstract}
Large phage antibody libraries are expected to be an efficient technology for obtaining humanized anti-tumor antibodies. However, few reports have been concerned about the selection strategies for intact cancer cells as targets. In this study, a $2 \times 10^{11}$ large naïve scFv library from blood B lymphocytes of normal adult donors and neonatal cord blood was constructed using the LoxP-cre system. Three human esophageal cancer cell lines were equally mixed for use as the target. These intact cells were divided into two groups, PF and NF, according to the treatment method of the cells (fixed with $2 \%$ PFA or not, respectively). Positive phage antibodies were identified following 4 panning cycles of adhesion, elution and amplification. Using a cell ELISA assay, it was found that the additional procedure of directly infecting XL1-Blue bacteria with the cell pellet following washing with an acidic solution can effectively decrease the loss of positive phage, yielding a positive screening rate of $11.6 \%$ (61/525). Most of the phage antibodies displayed binding activity with all three esophageal cancer cell lines. Moreover, other phages (such as NFc53a and NFc70a) appeared to be specific for certain cell lines. Regarding the method used to treat the target cells, both the positive screening rate and the genetic diversity of the antibody variable region were significantly higher in the NF group (12.9 and $71.4 \%$, respectively) than in the PF group (10.5 and $14.2 \%$, respectively). Immunohistochemical analysis demonstrated that the $\mathrm{scFv}$ phages have good specificity for esophageal cancer. This technology is helpful for developing small molecular tracers and targeted therapies for malignant tumors.
\end{abstract}

Correspondence to: Dr Xiaohang Zhao or Dr Dajin Zhang, Center for Basic Medical Sciences, Navy General Hospital of Chinese PLA, Beijing 100048, P.R. China

E-mail:xhztao@126.com

E-mail: dajinzhang@yahoo.com.cn

${ }^{*}$ Contributed equally

Key words: phage antibody library, scFv, LoxP-cre, cancer cell

\section{Introduction}

Monoclonal antibody technology is a very important tool in molecular biology, medicine, clinical diagnosis and treatment (1). However, murine monoclonal antibodies can cause serious immune responses in patients, which has led to the study of antibody humanization technologies (2). ReoPro is the first humanized antibody that was authorized for clinical treatment (3). The clinical data showed that its immunogenicity is far lower than that of murine antibodies. To further decrease the immune response, several humanized antibody transformation technologies have been successfully developed and applied (4). Phage antibody libraries are one of the important technological platforms used to obtain humanized antibodies. This technology assembles diverse variable region gene segments into an expression vector and fuses the molecular antibody fragments to a phage coat protein on the surface of phage particles to obtain the full repertoire of phage antibody diversity. Phage antibody libraries have powerful screening ability, as shown by their ability to integrate genotype and phenotype, to couple selection ability with expansion capacity, and mimic the in vivo antibody production process in vitro.

The construction technology and screening capacity of phage antibody libraries were markedly improved after the first phage combinatorial library was successfully constructed by Huse et al (5), which provided a new approach to obtaining such antibodies. Cancer therapy is the most active research field for antibody drugs. The cell membrane, being exposed to the outside environment, is an important site of a variety of cellular processes such as cell-cell contact, surface recognition, signal transduction, enzymatic activity and cytoskeleton contact, or for transporting substances across the membrane. Therefore, membrane proteins are absorbing targets for antibody drug in cancer therapy (6). For intact cancer cells as targets, the requirements for antibody library size and diversity are much higher due to the heterogeneity of cancer cells, their extremely complex surface antigens, and the uneven distribution and different levels of exposure of membrane proteins.

Currently, the strategy for anti-tumor antibody screening involves making a phage antibody library through the amplification of peripheral blood B lymphocytes derived from immunized animals or cancer patients (7). However, because 
of immune escape, cancer cells often fail to evoke a normal immune response and may even cause immune suppression, which results in B cell population changes and reduced antibody diversity. Thus, although there have been many related studies, anti-tumor antibodies with therapeutic activity have only rarely been reported.

A natural antibody library with a large size provides a better method for obtaining anti-tumor antibodies. Phage antibody libraries up to $10^{10}-10^{11}$ in size with good diversity can effectively provide each kind of humanized antibody. Their range of application has continually expanded to the screening of antibodies with multiple antigens, including whole cells, tissue sections, antigen blotted film and even living organs in vivo. Both the specificity and affinity of the screen can be precisely modulated. It is possible to obtain any specific humanized antibody without immunization by using a large antibody library. In this way, the low efficiency of $\mathrm{mAb}$ production and the instability of hybridomas can be overcome.

However, it is not easy to construct large phage antibody libraries that meet our requirements. When using phage display to construct a natural antibody library, diversity and library size are the important quality indices. In addition, the library size is proportional to the affinity of the antibodies (8). It is much easier to screen high-affinity antibodies when the library size is $>10^{10}(9,10)$. The biggest limitation of constructing a large antibody library is the low efficiency of bacterial transformation. To solve this problem, Griffiths et al (11) first constructed a large antibody library using light chain variable region (VL) and heavy chain variable region (VH) recombination mediated by the LoxP-cre recombination system. Recombinant sequences were designed in two vectors, and then the $\lambda$ light chain $(\mathrm{V} \lambda), \kappa$

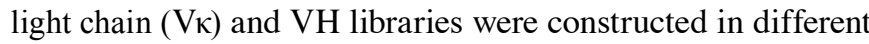
vectors, the light chain and heavy chains were changed by the LoxP-cre recombination system of phage P1 and finally a Fab phage antibody library with a size of $6.5 \times 10^{10}$ was constructed. However, this double vector system has the shortcomings of poor stability and high frequency of antibody gene deletion.

In this study, we constructed a large single chain antibody library by using LoxP-cre system-mediated recombination within a single vector, which was first reported by Sblattero and Bradbury (12). In this system, the antibody genes are inserted into a vector, and LoxP and LoxP511 sequences were designed to be upstream and downstream, respectively, of the heavy chain in this vector. The cre protein catalyzes LoxP- and LoxP511-specific recombination, but not that between LoxP and LoxP511, resulting in a wide diversity of genes reflecting the diversity of the light and heavy chain variable region combination. Compared with the earlier two-vector system, all of the clones in this system are functional. Moreover, both the recombination rate and the stability of the antibodies are improved. This clever method enhances the library size of the antibody library, reduces the workload of traditional construction and greatly increases the diversity of antibody genes, thereby providing the necessary and sufficient conditions for successful antibody screening.

In the current study, we constructed a $2 \times 10^{11}$ large natural antibody library from blood B lymphocytes of normal adult donors and neonatal cord blood by using the LoxP-cre system. Using this library to screen esophageal cancer cell lines, we obtained 5 different phage single chain antibodies that specifically recognize esophageal cancer cells. Furthermore, we investigated the screening conditions for whole cells. This technology is expected to be an effective tool for developing small molecular tracers and targeted therapies for malignant tumors.

\section{Materials and methods}

Bacterial stains, culture medium and reagents. $\mathrm{pDF}$ is a phage antibody expression vector based on pDNA5 (a gift from professor Bradbury) that was created by Professor Yan Wang (11). This vector can express either Fab or scFv (Fig. 1) and can be used for LoxP-cre site-specific recombination.

XL1-Blue (with tetracycline resistance) and helper phage VCSM13 were present in our laboratory. BS1365 [genotype: F'kan recA1 endA1 gryA96 thi21 $\Delta$ lacU169supE44 hsdR17 (imm434X12cre)] was kindly provided by Professor Sauer (The US Oklahoma Medical Foundation). Restriction enzymes and T4 DNA ligase were purchased from Fermentas and Promega, respectively. TaqDNA polymerase was purchased from the Shanghai Biological Engineering Company. The synthesis and sequencing of primers PDF3 and Leds were performed by the Beijing Liuhe Huada gene company. Human ovalbumin (OA) was purchased from Sigma. Human keratin (ker), human TNF- $\alpha$, human anti-tyrosinase related protein 1 (TRP-1), human interleukin 8 (IL-8), digoxin (Dig) and ricin were generated in our laboratory and collaborating laboratories. Horseradish peroxidase-labeled anti-mouse M13 antibody was obtained from Invitrogen. Immuno-tubes were purchased from Denmark NUNC. Microtiter plates and cell culture plates were products of Coster; human esophageal cancer cell lines KYSE-170 and KYSE-180 were gifts from Dr Y Shimada (University of Kyoto, Japan). EC0156 was from the State Key Laboratory of Molecular Oncology (13).

Primer design and primary library construction. pDF (Fig. 1) expresses small molecule Fab antibodies or scFv by its LoxP-cre site. This vector has a Lac promoter and a succinate terminator (TAG), and it has V5 and His tags connected with scFv. In non-succinate inhibition strains, translation of the polypeptide chain is terminated at the TAG, and soluble protein is formed under the guidance of the signal peptide. Wild-type LoxP is located downstream of $\mathrm{VH}$, and it has only one base (G was changed to A) different from the mutant LoxP511. By using the restriction sites $B s s \mathrm{HII}$ and NheI, the $\mathrm{scFv}$ gene can be inserted. To obtain variable $\mathrm{V}$ gene segments, B lymphocytes were collected from peripheral blood of 6 normal adult donors and neonatal cord blood of 10 donors. Peripheral blood lymphocytes (PBLs) were prepared and purified using lymphocyte separation medium. Following the preparation of total RNA, cDNA was synthesized by reverse-PCR, and a primer set was used to amplify light and heavy chain variable region genes, which in theory encompass the entire repertoire of human antibody genes (Table I). The different subtype populations (VL and VH segments) were found to be distributed proportionally to the normal germline variable region gene frequencies. A second PCR was conducted by using extension primers to obtain VL and VH fragments containing endonuclease sites. The scFv gene repertoire was obtained by overlapping PCR to splice VLs with VHs. Following digestion and ligation of scFv and pDF by BssHII and NheI, the plasmids with scFv genes 


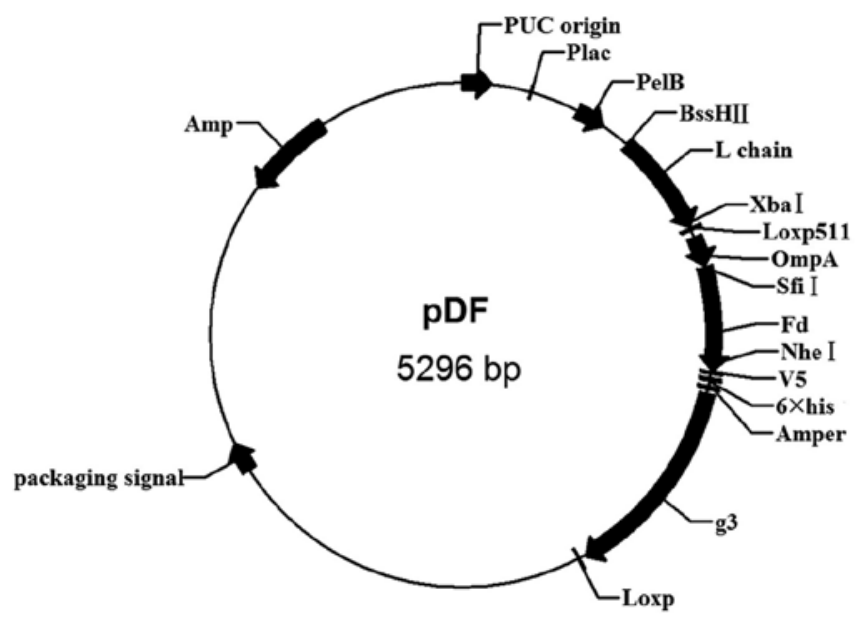

Figure 1. Map of the phagemid vector pDF used for the construction of the scFv phage library. This 5296 bp vector was modified from pDNA5, which was derived from M13. Transcription is under the control of the lac promoter. The ampicillin (Amp) resistance gene is used for the selection and maintenance of the phagemid. The phagemid carries the PUC origin of replication for amplification in phage and E. coli. PelB and OmpA are leader peptides that assist the expressed $\mathrm{scFv}$ and $\mathrm{Fab}$ into the bacterial periplasm. LoxP is located downstream of gene III, and LoxP511 is in between the light chain and heavy chain. The scFv genes were cloned between BssHII and NheI, and the Fab genes were cloned with BssHII + XbalI and Sfi + NheI for display on the phage coat protein $\mathrm{pIII}$. An in-frame amber stop codon permits the expression of scFv or Fab as a fusion with the 6xHis-tag and V5-tag in a non-suppressor host strain (HB2151). Using cre-recombinase, light chains and heavy chains of different phagemids can exchange with each other by recombination.

were sub-cloned into $E$. coli XL1-Blue by electroporation. The library size was calculated through culturing the appropriate bacteria in agar dishes $(100 \mu \mathrm{g} / \mathrm{ml}$ ampicillin). Plasmids were extracted to calculate the $\mathrm{scFv}$ recombination rate. The remaining bacteria were inoculated into $500 \mathrm{ml}$ of SB culture medium with VCSM13 helper phage (pfu, $1.7 \times 10^{12}$ ) for rescue and cultured overnight at $30^{\circ} \mathrm{C}$. The supernatant was collected, and the primary phage antibody library was obtained after PEG precipitation. The titer of the primary phage antibody library was determined.

Construction of a large antibody library through LoxP-cre recombination. The concentration of BS1365 bacteria constitutively expressing cre-recombinase (cultured in 2YT medium containing $50 \mu \mathrm{g} / \mathrm{ml}$ kanamycin and $10 \mathrm{~g} / 1$ glucose) was calculated at a logarithmic phase (A600, 0.5). BS1365 cells were superinfected with the primary phage antibody library [multiplicity of infection (MOI), 200] for $1 \mathrm{~h}$ at $37^{\circ} \mathrm{C}$ without shaking, allowing the multi-copy plasmid to infect the cells. 2YT medium was added to $400 \mathrm{ml}$, Amp was added to $100 \mu \mathrm{g} /$ $\mathrm{ml}$, and the cells were grown to A600, 0.5 with shaking after which, $5 \mathrm{ml}$ of VCSM13 helper phage (pfu, $1.7 \times 10^{12}$ ) was added, and growth continued for $1 \mathrm{~h}$ at $37^{\circ} \mathrm{C}$. The phage antibody library was then collected, and its titer was evaluated. One liter of logarithmic phase XL1-Blue $\left(5 \times 10^{8}\right.$ cells $\left./ \mathrm{ml}\right)$ bacteria was infected at a phage:bacteria ratio of $\leq 1(\mathrm{MOI} \leq 1)$ for $30 \mathrm{~min}$ at $30^{\circ} \mathrm{C}$. The library size was calculated in culture dishes containing Amp. Then, enough Amp and $10 \mathrm{ml}$ of VCSM13 were added, and growth continued at $30^{\circ} \mathrm{C}$ overnight. The supernatant was collected, and PEG precipitation was used to recover the large recombination phage antibody library. The titer was examined, and plasmid DNA was extracted for DNA fingerprinting analysis.

Solid-phase antigen screening of the phage antibody library. Immuno-tubes were coated with $1 \mathrm{ml}$ of $50-100 \mu \mathrm{g} / \mathrm{ml}$ antigen in $0.05 \mathrm{~mol} / \mathrm{l}$ carbonate buffer $\left(\mathrm{pH} \mathrm{9.6)}\right.$ at $4^{\circ} \mathrm{C}$ overnight, blocked with $2.5 \%$ milk and finally incubated in $1 \mathrm{ml}$ of phage antibody library (approximately $1 \times 10^{13} \mathrm{pfu}$, in $1 \% \mathrm{BSA} /$ PBS) for $2 \mathrm{~h}$ at $37^{\circ} \mathrm{C}$. After removing the phage solution and rinsing twice with PBST (the rinsing times were increased for each subsequent round) and once with distilled water, the solid phase attached phage antibodies were recovered using 2 methods. Fresh XL1-Blue bacteria $(1 \mathrm{ml})$ were first added and incubated for $15 \mathrm{~min}$ at $37^{\circ} \mathrm{C}$, which was then changed to $10 \mathrm{ml}$ of SB containing $20 \mu \mathrm{g} / \mathrm{ml} \mathrm{Amp} \mathrm{and} 10 \mu \mathrm{g} / \mathrm{ml}$ Tet. Finally, the post-infected immuno-tubes were washed with distilled water twice and eluted in $1 \mathrm{ml} 0.1 \mathrm{M}$ glycine buffer (containing $0.1 \%$ BSA, pH 2.2) for $15 \mathrm{~min}$ at $37^{\circ} \mathrm{C}$, and subsequently $40 \mu \mathrm{l}$ of $2 \mathrm{~mol} / \mathrm{l}$ Tris was added for neutralization. After adding $10 \mathrm{ml}$ of XL1-Blue cells and allowing to stand for $15 \mathrm{~min}$ at $37^{\circ} \mathrm{C}$, $10 \mathrm{ml}$ of SB (containing Amp and Tet) was added. Appropriate dilutions of bacteria from 2 cycles were used to examine $\mathrm{CFU}$, and the remaining bacteria were cultured for $4 \mathrm{~h}$ at $37^{\circ} \mathrm{C}$ in $50 \mathrm{ml} \mathrm{SB}$. Subsequently, $1-2 \times 10^{12}$ pfu helper phage VCSM13 was added, and the culture was shaken overnight at $30^{\circ} \mathrm{C}$. Phage particles were concentrated by $\mathrm{PEG} / \mathrm{NaCl}$, dissolved in PBS/BSA, and then used for a subsequent round of selection. The 2 nd, 3rd and 4 th rounds of panning were conducted similarly to this procedure. The titer of the eluted phage and the recovery rate were determined after each bio-screening, and these served as indices of specific phage antibody enrichment.

Esophageal cancer cell screening using the phage antibody library. Three human esophageal cancer cell lines, KYSE180, KYSE-170 and EC0156, all in good growth status, were digested with trypsin and washed twice in PBS. The intact cells were equally mixed to form a cell pool and then divided into 2 equal portions of approximately $4-5 \times 10^{7}$ cells each. The screen was performed in two rounds, PF and NF, according to the treatment method of the cells (fixed with $2 \%$ PFA or not). Before first round of panning against cancer cells, $4 \mathrm{ml}$ phage antibody library (an aliquot containing about $2 \times 10^{13} \mathrm{pfu}$ ) was pre-incubated with $8 \times 10^{7} \mathrm{~B}$ lymphocytes from the peripheral blood of healthy adult donors. Then each half of unbounded phages was incubated with cancer cells in PF and $\mathrm{NF}$ respectively for $2 \mathrm{~h}$ at $37^{\circ} \mathrm{C}$. The supernatant was removed by $1,000 \mathrm{rpm} / \mathrm{min}$ centrifugation, and the cells were washed in PBST 5 times to remove unbound phage (the washing times were increased for each round for a total of 4 rounds). After washing, the cells were incubated in $2 \mathrm{ml}$ of elution buffer for $15 \mathrm{~min}$ at $37^{\circ} \mathrm{C}$ followed by the addition of $80 \mu \mathrm{l}$ of $2 \mathrm{~mol} / \mathrm{l}$ Tris neutralizing solution. Two portions, supernatant (s) and cell pellet (c), were obtained after centrifugation. Each portion was infected with $2 \mathrm{ml}$ of XL1-Blue for $15 \mathrm{~min}$, and the titer of the eluted phages was examined. The bacteria were cultured for $3 \mathrm{~h}$ at $37^{\circ} \mathrm{C}$ in $10 \mathrm{ml}$ of $\mathrm{SB}$ and were then propagated overnight at $30^{\circ} \mathrm{C}$ in $100 \mathrm{ml}$ of SB with $1.7 \times 10^{12} \mathrm{pfu}$ VCSM13 helper virus. The first round of enriched phages were harvested and used for the next round of screening, as described above. 
Table I. Primers used to construct the scFv library.

\begin{tabular}{ll}
\hline Name & \multicolumn{1}{c}{ Sequence } \\
\hline Forward primers for VH & \\
PVH1 & TATCCTCGAGCGTACCSAGGTSCAGCTGGTRCAGTCTGG \\
PVH2 & TATCCTCGAGCGTACCSAGGTGCAGCTGKTGGAG \\
PVH3 & TATCCTCGAGCGTACCCAGRTCACCTTGAAGGAGTCT \\
PVH4 & TATCCTCGAGCGTACCCAGSGCAGCTRCAGSAGT
\end{tabular}

Reverse primers for $\mathrm{VH}$

PVHM

GGCGGATGC $\underline{G C T A G C T G A R G A G A C R G T G A C C}$

Extended forward primer for $\mathrm{VH}$,

used for overlap PCR

PVHL

CCATAACTTCGTATAATGTATACTATACGAAGTTATCCTCGAGCGGTACC

Forward primers for $\mathrm{V} \kappa$

HVK1

HVK2

HVK3

HVK4

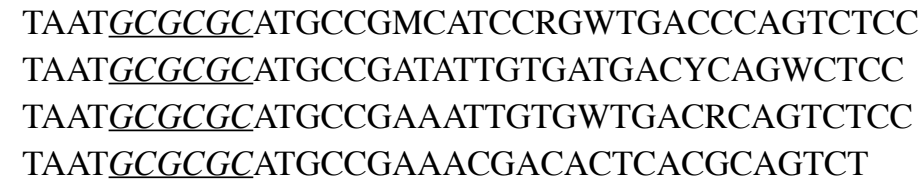

Forward primers for $\mathrm{V} \lambda$

HVL1

HVL2

HVL3

HVL4

HVL5

HVL6

HVL7

Extended forward primer for $\mathrm{V} \kappa, \mathrm{V} \lambda$

HVKEX

\author{
TAATGCGCGCATGCCCAGTCTGTGYTGACKCAGCC \\ TAAT GCGCGCATGCCCAGTCTGCCCTGACTCAGCC \\ TAATGCGCGCATGCCTCCTMTGAGCTGACWCAG \\ TAATGCGCGCATGCCCAGTYTGTGCTGACTCAATC \\ TAATGCGCGCATGCCCAGRCTGTGGTGACYCAGGAG \\ TAATGCGCGCATGCCAATTTTATGCTGACTCAG \\ TAAT $\underline{G C C G C A T G C C C A G K M T G R G C T G A Y G C A G C C A C C C T C}$
}

GAGGAGGAGATAAT $\underline{G C G C G C A T G C C}$

GGTCGACCCTCCGGAACGTTTGATHTCCASCTTGGT

GGTCGACCCTCCGGAACGTTTAATCTCCAGTGCTGT

GGTCGACCCTCCGGAACCTAGGACGGTSAGCTTGGTCCC

Reverse primers for $\mathrm{V} \kappa$

PVK1

PVK2

Reverse primer for $\mathrm{V} \lambda$

PVL

Extended reverse primer for VL,

used for overlap PCR

PVLEX

GGATAACTTCGTATAGTATACATTATACGAAGTTATGGTCGACCCTCCGGAAC

The primers are complementary to the V-gene segments. Four pairs of heavy chain variable region (VH) primers, 4 pairs of the $\kappa$ light chain $\mathrm{V} \kappa$ primers and 7 pairs of the $\lambda$ light chain $\mathrm{V} \lambda$ primers were designed, which can amplify a full set of antibody variable region gene repertoires. To be able to use either $\mathrm{scFv}$ or Fab forms to construct antibody libraries, we retained IgM CH1 in the first round amplification primers, and we added the restriction sites Bss HII (GCGCGC) and NheI (GCTAGC) to the second round amplification primers (sequences indicated in italics and underlined). Each extension primer (PVHL and PVLEX) has a 38 base overlap (as a linker containing the LoxP511 sequence (underlined)) and connects VH and light chain variable region (VL) to form scFv by overlap PCR. The traditional sequence (G4S) 3 was replaced by LoxP511, which is shorter, flexible and has the ability to prevent proteolysis.

ELISA assay to identify the specifically bound phage antibodies. Individual phage clones were randomly selected after the 4th round of screening from culture plates and cultured in $1 \mathrm{ml}$ of SB with VCSM13 helper virus overnight at $30^{\circ} \mathrm{C}$. The supernatant with $\mathrm{scFv}$ phage was collected for ELISA assays according to the protocol. Microtiter plates were coated with
$50 \mu \mathrm{l} /$ well $10 \mu \mathrm{g} / \mathrm{ml}$ antigen in carbonate buffer for $2 \mathrm{~h}$. After blocking with $2.5 \%$ skim milk, scFv phages were added into the ELISA plates and incubated for $1 \mathrm{~h}$ at $37^{\circ} \mathrm{C}$. The plates were washed with $0.05 \%$ Tween-20-PBS buffer 3 times. Bound phages were detected with HRP-labeled mouse anti-M13 monoclonal antibody at 1:5,000. The plates were then washed 
Table II. Screening and identification of antibodies in the library using 7 pure antigens.

\begin{tabular}{|c|c|c|c|c|c|c|}
\hline \multirow[b]{2}{*}{ Antigen } & \multicolumn{3}{|c|}{ Positive rate } & \multicolumn{3}{|c|}{ Diversity } \\
\hline & $\begin{array}{c}\text { Total clones } \\
\text { (A) }\end{array}$ & $\begin{array}{l}\text { Positive clones } \\
\text { (B) }\end{array}$ & $\begin{array}{c}\mathrm{B} / \mathrm{A}(\%) \\
(\mathrm{C})\end{array}$ & $\begin{array}{l}\text { Total clones } \\
\text { (E) }\end{array}$ & $\begin{array}{l}\text { Different clones } \\
\text { (F) }\end{array}$ & $\begin{array}{c}\mathrm{F} / \mathrm{E}(\%) \\
(\mathrm{G})\end{array}$ \\
\hline $\mathrm{OA}$ & 148 & 17 & 11.5 & 17 & 11 & 64.7 \\
\hline ker & 48 & 16 & 33.3 & 16 & 9 & 56.2 \\
\hline TNF- $\alpha$ & 85 & 14 & 16.5 & 14 & 5 & 35.7 \\
\hline TRP-1 & 50 & 18 & 36 & 18 & 3 & 16.7 \\
\hline IL-8 & 60 & 2 & 3.3 & 2 & 2 & 100 \\
\hline Dig & 80 & 16 & 20 & 16 & 10 & 62.5 \\
\hline Ricin & 250 & 88 & 35.2 & 40 & 7 & 17.5 \\
\hline
\end{tabular}

After 3-4 rounds of panning, clones (A) were selected for ELISA identification. Specific phage clones were calculated (B); (C) is the positive rate of each screen. The diversity of the $\mathrm{scFv}$ genes was examined by DNA fingerprint analysis. Plasmids of positive clones (E) were extracted, and their $\mathrm{scFv}$ genes were amplified. PCR products were digested by the restriction enzyme $M v a \mathrm{I}$ at $37^{\circ} \mathrm{C}$ for $3 \mathrm{~h}$. PAGE (6\%) was performed, and the number of clones with different DNA fingerprints $(\mathrm{F})$ was determined.

in $0.05 \%$ Tween-20-PBS buffer, and OPD substrate was added. The absorbance at $490 \mathrm{~nm}$ was analyzed on an ELISA plate reader. For cell ELISA, equal numbers $\left(5 \times 10^{4}\right.$ cells/well $)$ of KYSE-180, KYSE-170 and EC0156 cells were seeded on the same 96 -well plate at $37^{\circ} \mathrm{C}$ for $1 \mathrm{~h}$, with empty wells as controls. The supernatant was removed after centrifugation, and the cells were fixed with 4\% PFA for 30 min and then blocked with $2.5 \%$ skim milk. The phage antibody was adjusted to $1 \times 10^{11}$ cfu as the first antibody, and HRP-labeled mouse anti-M13 monoclonal antibody at a dilution of 1:1,000 was used as the secondary antibody. Anti-OA scFv phage was used as the negative control. The other cell ELISA procedure was the same as the phage ELISA described above.

Immunochemical staining. KYSE-180, KYSE-170 and EC0156 cells were seeded on coverslips, cultured for $24 \mathrm{~h}$ and then fixed with 4\% PFA for $30 \mathrm{~min}$ at room temperature. Triton X-100 $(0.1 \%)$ was added to perforate the cells at room temperature for 5-10 min. After blocking for $2 \mathrm{~h}$ with $5 \%$ bovine serum, the phage antibody $\left(5 \times 10^{12} \mathrm{cfu}\right)$ was added, and the cells were incubated for $1 \mathrm{~h}$ at $37^{\circ} \mathrm{C}$ and detected with HRP-M13 antibody (1:100). The location of the phage was observed by microscope after DAB staining. For immunohistochemical analysis, $5 \mu \mathrm{m}$ thick serial sections cut from paraffin-embedded esophageal cancer tissues were deparaffined and rehydrated in gradient ethanol. Endogenous peroxidase was eliminated by treating the slides with $3 \% \mathrm{H}_{2} \mathrm{O}_{2}$. Antigen retrieval was performed in boiled sodium citrate buffer. The phage antibody was diluted to $1-5 \times 10^{13} \mathrm{cfu}$ and used as the first antibody. The following steps were similar to those above.

DNA fingerprinting analysis. scFv genes were amplified from phagemids of positive phage clones. The PCR products were purified by centrifugation with CL-6B gel microcolumns (Pharmacia) and digested with the restriction enzyme $M v a \mathrm{I}$ for $3 \mathrm{~h}$ at $37^{\circ} \mathrm{C}$ in the following $20 \mu \mathrm{l}$ reaction: DNA $10 \mu \mathrm{l}, \mathrm{MvaI}$ $1 \mu \mathrm{l}, 10 \mathrm{X}$ Buffer $2 \mu \mathrm{l}$ and $\mathrm{H}_{2} \mathrm{O} 7 \mu \mathrm{l}$. The restriction patterns were analyzed using $6 \%$ polyacrylamide gel electrophoresis
(PAGE). After ethidium bromide (EB) staining, the gels were observed, and photos were taken under UV light.

\section{Results}

Construction of a large phage antibody library. To ensure a diversity of $\mathrm{scFv}$ segments in the antibody libraries, $2 \times 10^{9}$ PBLs were collected from peripheral blood from 6 normal adult donors and neonatal cord blood from 10 donors. Of the $20 \mathrm{mg}$ of total RNA that was extracted, $200 \mu \mathrm{g}$ was reverse transcribed to cDNA. The $\mathrm{VH}, \mathrm{V} \kappa$ and $\mathrm{V} \lambda$ genes were amplified and then mixed according to the usage frequency of the different subtypes in normal people with a full repertoire of germline B genes. The scFv gene repertoires were obtained by overlapping PCR and then inserted into the pDF vector. The size of the primary library was measured at $1 \times 10^{7} \mathrm{cfu}$. The efficiency of functional clones in the phage library was approximately $86 \%$, as analyzed by digestion and electrophoresis of the $\mathrm{scFv}$ fragments. Following superinfection into BS1365 bacteria to induce the recombination of light and heavy chains by the LoxP-cre system, a $2 \times 10^{11}$ large phage antibody library was obtained after low MOI into XL1-Blue cells. The scFv gene recombination rate was $92 \%(13 / 14)$, as determined by DNA fingerprint analysis.

Identification of library antibodies using pure antigens. Seven antigens, including human OA, ker, TNF- $\alpha$, TRP-1, IL-8, Dig and ricin were used as targets for identifying specific antibodies in the library we constructed. After 3-4 rounds of screening, when the output/input ratio of the phages was increased, positive phage clones were identified by ELISA, and the diversity of the ScFv genes was examined by DNA fingerprint analysis. The average positive rate and diversity of these 7 antigens were 23.7 and $38.2 \%$, respectively (Table II).

Screening for anti-esophageal cancer cell phage antibodies. To exclude potential bias caused by differences between cell lines and to obtain anti-tumor antibodies with wide specificity, 
Table III. Recovery of phage binding to esophageal carcinoma cells in each round of selection.

\begin{tabular}{lccc}
\hline Round & Input phage $(\mathrm{pfu})$ & Output phage $(\mathrm{pfu})$ & Recovery rate $(\%)$ \\
\hline Group PF & & & $2.2 \times 10^{-4}$ \\
1 & $1.0 \times 10^{13}$ & $2.2 \times 10^{7}$ & $3.6 \times 10^{-5}$ \\
2 & $1.4 \times 10^{13}$ & $5.0 \times 10^{6}$ & $2.4 \times 10^{-6}$ \\
3 & $7.4 \times 10^{13}$ & $1.8 \times 10^{6}$ & $1.3 \times 10^{-5}$ \\
4 & $3.0 \times 10^{13}$ & $3.9 \times 10^{6}$ & $5.6 \times 10^{-4}$ \\
Group NF & & & $7.0 \times 10^{-5}$ \\
1 & $1.0 \times 10^{13}$ & $5.6 \times 10^{7}$ & $1.8 \times 10^{-6}$ \\
2 & $3.0 \times 10^{12}$ & $2.0 \times 10^{6}$ & $3.0 \times 10^{-6}$ \\
3 & $1.0 \times 10^{14}$ & $1.8 \times 10^{6}$ & $8.9 \times 10^{5}$ \\
\hline
\end{tabular}

The screening was performed in two groups, PF (fixed with PFA) and NF, (not fixed in PFA) according to the treatment method of the cells. The recovery rate was calculated as the output phage titer to input phage titer ratio after each round of selection. The washing strength was increased by 5 additional washes each round.

Table IV. Rates of positive antibody identification for fixed cells vs. living cells.

\begin{tabular}{lcrr}
\hline Method & Analyzed clones (A) & Positive clones (B) & B/A (\%) \\
\hline NFc & 130 & 12 & 9.2 \\
NFs & 119 & 20 & 16.8 \\
PFc & 128 & 6 & 4.7 \\
PFs & 148 & 23 & 15.5 \\
Total & 525 & 61 & 11.6 \\
\hline
\end{tabular}

The antibody library was incubated with the cells in the NF (not fixed in PFA) or PF (fixed with PFA) group for $2 \mathrm{~h}$, which were then washed with $0.05 \% \mathrm{PBS} /$ Tween. After glycine elution and centrifugation, bound phage particles were rescued by infecting XL1-Blue cells with the supernatant (s) and the cell pellet (c). The absorption-elution-amplification procedure was repeated 4 times, after which individual clones were analyzed for specific antigen binding by cell ELISA. B/A, raio of positive clones (B) over the analyzed clones (A).

three esophageal cancer cell lines, KYSE-170, KYSE-180 and EC0156, were equally mixed to form a cell pool. These intact cells were divided into two groups, PF and NF, according to the treatment method of the cells (fixed with PFA or not, respectively). The panning conditions of the two groups were the same. The recovery rate of binding phages in the 2 nd and 3 rd rounds of screening was lower because of the increased washing strength. However, in 4th round the recovery rate was 5.4-fold and 1.7-fold higher than in the 3rd round in PF and NF groups, respectively, indicating enrichment for phages specifically binding to esophageal carcinoma cells in both groups (Table III).

The binding activity of the phage antibodies to the esophageal cancer cells was determined by cell ELISA. All of the experiments were repeated more than 3 times. Positive clones were identified when A490>0.5, which is 3-fold higher than the negative control. No positive clones were found in the $3 \mathrm{rd}$ round among 100 clones (50 for each group). However, 61 positive clones were identified from 525 clones in the 4 th round (the average positive rate is $11.6 \%$ ). The positive rate in the NF group $(12.9 \%, 32 / 249)$ was much higher than that in the $\mathrm{PF}$ group $(10.5 \%, 29 / 276)$ (Table IV).
To rescue the esophageal carcinoma cell-binding phage antibodies, glycine elution was performed. The cell suspension was centrifuged, and the eluted phages were located in the supernatant (s). In addition, considering that there may have been some phages not removed from the cells by the acid elution buffer which thus remained on the surface of the cell pellet (c), both the supernatants (PFs and NFs) and the cell pellets ( $\mathrm{PFc}$ and $\mathrm{NFc}$ ) were used to infect XL1-Blue cells for amplification. The positive rates of the PFs and NFs groups were higher than those of the PFc and NFc groups, suggesting that most of the phages were washed off the cells by the glycine elution. However, a small portion of the bound phages were still left on the cell surface. We were able to rescue this portion of the phage antibodies by using the additional step of infecting XL1-Blue cells with the cell pellets (Table IV). The ratio of positive clones was higher in the NF groups (NFs and NFc) than in the PF groups (PFs and $\mathrm{PFc}$ ), and the NFs group showed the highest rate (16.8\%), whereas the PFc group showed the lowest (4.7\%).

Fourteen positive clones were selected for analysis of their variable region gene by DNA sequencing. Five clones with different antibody variable region gene subtypes (light or heavy chain) were obtained by analyzing the IgBLAST 
Table V. Comparison of positive clone diversity between fixed cell screening and living cell screening.

\begin{tabular}{|c|c|c|c|c|c|c|}
\hline \multirow[b]{2}{*}{ Method } & \multicolumn{3}{|c|}{ Sequencing } & \multicolumn{3}{|c|}{ DNA fingerprinting } \\
\hline & $\begin{array}{c}\text { Differential } \\
\text { clones (A) }\end{array}$ & $\begin{array}{c}\text { Total } \\
\text { clones (B) }\end{array}$ & $\mathrm{A} / \mathrm{B}(\%)$ & $\begin{array}{c}\text { Differential } \\
\text { clones }\end{array}$ & $\begin{array}{c}\text { Total } \\
\text { clones }\end{array}$ & $\mathrm{A} / \mathrm{B}(\%)$ \\
\hline NF & 5 & 7 & 71.4 & 5 & 11 & 45.5 \\
\hline $\mathrm{PF}$ & 1 & 7 & 14.3 & 2 & 9 & 22.2 \\
\hline Total & 5 & 14 & 35.7 & 6 & 20 & 30.0 \\
\hline
\end{tabular}

Fourteen clones were selected for DNA sequencing and analyzed by NCBI Ig-BLAST to determine their germline V-gene segments. The other $20 \mathrm{scFv}$ gene fragments were amplified by PCR and analyzed by DNA fingerprinting. Positive clones in the NF group showed more diversity than that in the PF group with both methods. A/B, ratio of differential clones (A) over total clones (B).

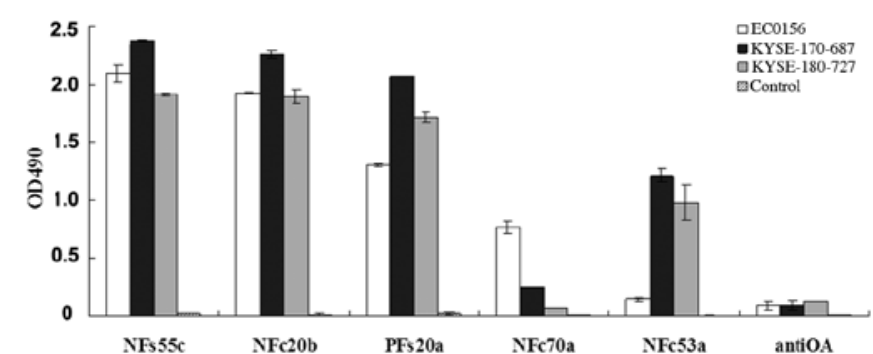

Figure 2. Binding activity of recovered clones by cell ELISA. Five of 61 positive phage clones selected by bio-panning against 3 different esophageal carcinoma cell lines. KYSE-170, KYSE-180 and EC0156 were coated onto a 96-well plate at $5 \times 10^{4}$ cells/well and fixed with $4 \%$ PFA before being incubated with representative phage adjusted to $1 \times 10^{11} \mathrm{cfu}$. Bound phages were detected with an anti-M13 phage-horseradish peroxidase (HRP) conjugate $(1: 1,000)$ using OPD as the substrate and detection at OD490. An irrelevant antibody (anti-OA phage $\mathrm{scFv}$ ) was used as the negative control. Three independent experiments were conducted, each with two parallel wells.

immunoglobulin gene database of NCBI. The gene sequences were the same among the 7 clones in the PF group, and the diversity rate was $14.2 \%(1 / 7)$. By contrast, 5 clones with different $\mathrm{V}$ genes were identified in the NF group for a diversity rate of $71.4 \%(5 / 7)$, and one of these had the same sequence as clones in the PF group. This finding suggests that the living cell screening method not only retains the epitopes of fixed cells but also makes it easy for the phage antibodies to capture more antigen epitopes. Another 20 positive clones were selected randomly for DNA fingerprint analysis of their $\mathrm{scFv}$ genes. The results revealed that the diversity rate of the NF group $(45.5 \%, 5 / 11)$ was much higher than that of the PF group $(22.2 \%, 2 / 9)$ (Table V).

Assessment of binding activity between the phage antibodies and esophageal cells. The binding activity of the phage antibodies with KYSE-170, KYSE-180 and EC0156 cells was examined by cell ELISA. Equal cell numbers of each cell line were coated onto the wells of a single 96-well plate with empty wells serving as controls. An anti-OA scFv phage was used as the negative control. Most of the 61 positive $\mathrm{scFv}$ phage clones showed binding activity with all 3 types of esophageal cells. However, a few showed cell-line specificity; for example, the ELISA signal of NFc53a for KYSE-170 cells was 8.5-fold higher than for EC0156 cells. Moreover, the ELISA signal of
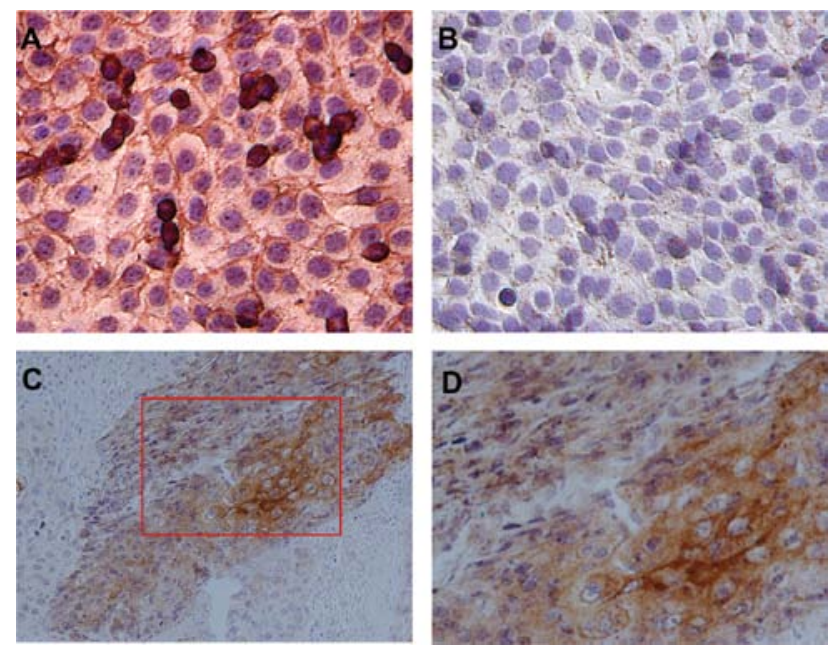

Figure 3. Immunochemical analysis of phage scFv NFc20b. Staining of KYSE-170 cells (A and B) and esophageal squamous cell carcinoma tissue slices (C and D) was performed with the selected phage scFv NFc20b. The bound phage was detected with horseradish peroxidase-conjugated anti-M13 (1:100) and DAB substrate. Cells were stained with NFc20b (A) or an irrelevant anti-OA phage $\mathrm{scFv}$ (B). Representative images are shown x200 (A, B and C) or $\mathrm{x} 400$ (D as a part of C).

NFc70a for EC0156 cells was 10.3-fold higher than for KYSE170 cells. The ELISA identification results for the 5 positive clones with different variable region genes are shown in Fig. 2.

The tissue specificity of the 5 positive phage antibodies with different variable region genes was assessed by immunochemical staining. Five of the phage antibodies showed binding activity with all 3 cancer cell lines to different extents. Positive staining was mainly detected in the cell membrane, with a low intensity staining in the cytoplasm. Positive staining was observed in esophageal tumor tissues, but not in adjacent tissues or interstitial cells, suggesting the tumor specificity of this phage antibody. More than 3 esophageal tumor tissues were evaluated by immunohistochemical analysis for each phage antibody. The results of the immunohistochemical analysis of $\mathrm{NFc} 20 \mathrm{~b}$ are shown in Fig. 3.

\section{Discussion}

Phage antibody libraries are an important technological platform for obtaining humanized antibodies. This technology can 
display small molecules, such as Fab, scFv, CDR3 and Fv, on the surface of phage particles and thereby capture the diverse repertoire of phage antibody (14). Small molecule antibodies have many advantages, including high tissue penetration capacity, low immunogenicity and fast serum clearance. Due to the deletion of their Fc segment, the molecules can provide low background radioimmunoimaging, and they are very useful molecules for drugs, toxins and radionuclides in targeted cancer therapy.

Although in theory a phage antibody library more than $10^{8}$ in size would meet the needs of most antigen screens, the success rate of identifying high-affinity antibodies from a phage antibody library is closely related to the library size. For some complex antigens, intact cancer cells for example, antibody screening requires a higher quality antibody library and a tighter screening process because most cell surface antigens have a specific conformation with glycosylation and other post-translational modifications. Cell surface antigens are much closer to the natural state than are purified antigens, and it is much easier to identify anti-tumor antibodies with actual applications. However, the composition of the cell surface is very complex, with many types of proteins, carbohydrates and lipids, but with a low number of tumor-specific antigens. Non-specific adhesion and loss of specific ligand are among the problems often faced when intact cancer cells are used as the target to screen an antibody library (15). Under these conditions, an antibody library of high diversity and library size is particularly important.

Depending on the source of antibody genes, a large antibody library can be constructed as semi-synthetic, synthetic or human lymphoid cell-derived. All of these have proven to be feasible methods $(8,16)$. The semi-synthetic and full synthetic methods require the synthesis of random CDR3 sequences. The length of the CDR3 heavy chain may vary from 5-30 amino acid residues, which induces synthesis difficulties. Natural libraries are advantageous because they are not limited by this length. Some have worried that it may be difficult to obtain self-antigen antibodies from a natural library because of the immunological tolerance to human autoantigens. According to reports, such worries have proven to be unnecessary (8). In contrast, compared to using peripheral blood B lymphocytes derived from immunized animals or cancer patients to construct an antibody library, natural antibody libraries avoid the diversity defects caused by immune escape of cancer cells when screening for cancer cell antibodies. To reduce the B lymphocyte diversity reduction caused by the adult acquired immune response, the B lymphocytes used in this study to amplify antibody variable region genes were derived not only from healthy adults but also from 10 samples of neonatal cord blood. This process ensured a diverse selection of antibody genes in the large antibody library and allowed for the identification of complex and varied antigens.

A natural phage antibody library up to $10^{10}-10^{11}$ in size with good diversity can effectively yield humanized antibodies for almost all target antigens. However, only few research groups can construct large phage antibody libraries because their construction requires a long laborious lawsuit and is very difficult. The application of the LoxP-cre site-specific recombination system in the construction of the antibody library can overcome the limitations of the poor transformation efficiency of $E$.coli to a certain extent. This system increases the matching and combination of light and heavy chain variable regions to construct large antibody libraries with high efficiency. Because phage titers significantly decrease after repeated freezing and thawing, a traditional phage antibody library cannot be effectively preserved for long periods. However, the recombination process can be repeated indefinitely by using the LoxP-cre recombination system. To a certain extent, this method relieves the preservation problem. The products of each recombination may have different diversity and can act as a substrate for the next recombination, which is conducive to antibody affinity maturation and constructing a specific antibody library. Sblattero and Bradbury (12), using in vivo recombination by a single recombinant vector, successfully constructed a single chain antibody library of $3 \times 10^{11}$ in size, showing greatly improved recombinant rates and stability compared to those of the two vector systems. However, few studies have reported that the technology was successfully used for the construction of a library, and the library size is only approximately $1-2 \times 10^{10}(17)$.

In this study, we used LoxP-cre system-mediated single cell recombination to construct a natural phage antibody library of $2 \times 10^{11}$ in size. The pDF vector was constructed in our lab based on pDNA5, and it can be used to construct both scFv and Fab libraries. To ensure the diversity of antibody genes, we harvested $4 \times 10^{9} \mathrm{~B}$ lymphocytes from both healthy people and cord blood. We obtained $20 \mathrm{mg}$ of total-RNA and used $200 \mu \mathrm{g}$ of for the reverse transcription based on the principle that each B cell can produce 100 Ig mRNA molecules. All of the reverse transcription products were used for PCR, and the diversity of $\mathrm{B}$ cells should be approximately $10^{8}$. Primers that can amplify the variable region genes of all the germline antibodies were designed. The products of different primers were mixed according to the usage frequency of the variable region genes in the human germline. The size of the primary library is $10^{7}$, and it can produce a diversity of $10^{14}$ in theory after random light and heavy chain matching mediated by the LoxP-cre specific-site recombination system. We obtained an antibody library with a general size of $10^{10}-10^{11}$ (per $1,000 \mathrm{ml}$ bacterial culture) after recombination. Thus, the library constructed after the recombination can have greater diversity, and it can provide a reliable platform for antibody screening. The large antibody library was verified by screening of 7 kinds of antigens, including cytokines (TNF- $\alpha$ and IL-8), a toxin protein (ricin) and a small molecule (Dig).

Screening strategies are critical for the biological efficiency of humanized single chain antibody libraries (18). Although most previous studies have used non-fixed living cells as the targets to screen for cancer cell antibodies $(7,19,20)$, some researchers still prefer to use PFA fixed cells to screen for antibodies. Fixed cells ensure the integrity of the cell membrane during the entire process $(21,22)$, decrease cell breakage and antigen degradation and avoid the inhibition of bacterial growth caused by hydrolytic enzymes and other harmful substances released by the breakdown of residual cancer cells. However, the process of fixation may prevent exposure of the antigen epitopes and cause tumor-specific antigens to be lost. In this study, we used the PF and NF screening methods side by side, and we found that even when cells were treated with a low concentration of fixative solution (2\% PFA), the screening 
efficiency was much better in the NF group than the PF group. Moreover, the variable region gene diversity of the positive antibodies in the NF group $(71.4 \%, 5 / 7)$ was also significantly higher than that in the PF group $(14.2 \%, 1 / 7)$. These data suggest that the living cell screening method not only retains the epitopes of fixed cells but also makes it easy for the phage antibodies to capture more antigen epitopes.

In the antibody library screening process, glycine, triethylamine and thiocyanate elution were usually used to recycle the bound phages, the concentration of which can be appropriately adjusted to improve the strength of the elution and thereby obtain high-affinity antibodies. However, in some cases, the interaction between antigen and antibody could not be dissociated by acid elution. Thus, some researchers have advocated the use of direct bacterial infection to avoid the loss of phage antibodies during the recycling process. In our laboratory, we have successfully recovered pure antigen antibodies by directly infecting bacteria with phages bound on the surface of antigens without acid elution. However, in the process of direct infection by bound phages to intact cancer cells, when the ratio of positive bacteria to cells is less than 1:25, the growth and proliferation of the positive bacteria are significantly inhibited by broken cancer cells (data not shown). In the current study, we first used glycine elution $(\mathrm{pH} 2.2)$ to elute and recycle the bound phages (s group), and we then used the cancer cells to directly infect the proliferative bacteria (c group), which decreased the loss of specific antibodies by a large extent.

The cancer cells used in this study were from esophageal cell lines established from primary human esophageal tumor tissues. To rule out bias due to individual differences in esophageal cancer patients and to obtain antibodies with wide spectrum of anti-tumor activity, we made a cell pool with an equal mixture of three esophageal cancer cell lines (KYSE-170, KYSE-180 and EC0156) and used the intact cells as the target. The results revealed that most of the positive phage antibodies showed binding activity with all 3 esophageal cancer cell lines, and a few clones, such as NFc53a and PFs127b, showed cell line specificity. Immunohistochemical results showed that 5 phage antibodies with different variable region gene sequences specifically recognize esophageal tumor tissues, but not adjacent tissues or interstitial cells, suggesting that their binding is quite tumor-specific.

In summary, we constructed a large humanized phage antibody library and investigated different strategies for identifying intact cancer cell single chain antibodies. The large antibody library is not only useful for finding tumor-specific antigens but also provides a platform for the screening of highly sensitive and specific anti-tumor antibodies. For example, specific antibodies screened from a single cancer cell can be used to identify rare peripheral blood cancer cells for the early diagnosis of tumorigenesis and the real-time monitoring of tumor recurrence and metastasis. $\mathrm{scFv}$ is easily manipulated by genetic technologies, and it can be modified to diabody and minibody multivalent small molecular antibodies $(6,23)$. Compared with intact antibodies, multivalent small molecular antibodies have shown the best balance of tumor targeting, tissue penetration and blood clearance rate. Thus, they are a promising vehicle for immunotherapy (24-27), and they may play important roles in site-specific imaging diagnosis and targeted tumor therapy.

\section{Acknowledgements}

We warmly thank professor Yan Wan for his suggestions on antibody library construction and antibody screening. This study was supported in part by the National Natural Science Foundation of China (nos. 31071256; 91029725; 30973373) and the Innovation Fund of the Navy General Hospital of Chinese PLA (no. CX200904).

\section{References}

1. Nelson AL and Reichert JM: Development trends for therapeutic antibody fragments. Nat Biotechnol 27: 331-337, 2009.

2. Vaughan TJ, Osbourn JK and Tempest PR: Human antibodies by design. Nat Biotechnol 16: 535-539, 1998.

3. Brekke OH and Loset GA: New technologies in therapeutic antibody development. Curr Opin Pharmacol 3: 544-550, 2003.

4. Reichert JM: Monoclonal antibodies as innovative therapeutics. Curr Pharm Biotechnol 9: 423-430, 2008.

5. Huse WD, Sastry L, Iverson SA, et al: Generation of a large combinatorial library of the immunoglobulin repertoire in phage lambda. Biotechnology 24: 517-523, 1992.

6. Imai S, Nagano K, Yoshida Y, et al: Development of an antibody proteomics system using a phage antibody library for efficient screening of biomarker proteins. Biomaterials 32: 162-169, 2011.

7. Xu MY, Xu XH, Chen GZ, et al: Production of a human singlechain variable fragment antibody against esophageal carcinoma. World J Gastroenterol 10: 2619-2623, 2004.

8. Sheets MD, Amersdorfer P,Finnern R, et al: Efficient construction of a large nonimmune phage antibody library: the production of high-affinity human single-chain antibodies to protein antigens. Proc Natl Acad Sci USA 95: 6157-6162, 1998.

9. Vaughan TJ, Williams AJ, Pritchard K, et al: Human antibodies with sub-nanomolar affinities isolated from a large non-immunized phage display library. Nat Biotechnol 14: 309-314, 1996.

10. de Haard HJ, van Neer N, Reurs A, et al: A large non-immunized human Fab fragment phage library that permits rapid isolation and kinetic analysis of high affinity antibodies. J Biol Chem 274: 18218-18230, 1999.

11. Griffiths AD, Williams SC, Hartley $\mathrm{O}$, et al: Isolation of high affinity human antibodies directly from large synthetic repertoires. EMBO J 13: 3245-3260, 1994.

12. Sblattero D and Bradbury A: Exploiting recombination in single bacteria to make large phage antibody libraries. Nat Biotechnol 18: 75-80, 2000.

13. Wang Q, Xu Y, Zhao X, et al: A facile one-step in situ functionalization of quantum dots with preserved photoluminescence for bioconjugation. J Am Chem Soc 129: 6380-6381, 2007.

14. Pande J, Szewczyk MM and Grover AK: Phage display: concept, innovations, applications and future. Biotechnol Adv 28: 849-858, 2010.

15. Kurosawa G, Sumitomo M, Ukai Y, et al: Selection and analysis of anti-cancer antibodies for cancer therapy obtained from antibody phage library. Cancer Sci 102: 175-181, 2011.

16. Hodits RA, Nimpf J, Pfistermueller DM, et al: An antibody fragment from a phage display library competes for ligand binding to the low density lipoprotein receptor family and inhibits rhinovirus infection. J Biol Chem 270: 24078-24085, 1995.

17. Cen X, Bi Q and Zhu S: Construction of a large phage display antibody library by in vitro package and in vivo recombination. Appl Microbiol Biotechnol 71: 767-772, 2006.

18. Popkov M, Rader C and Barbas CF III: Isolation of human prostate cancer cell reactive antibodies using phage display technology. J Immunol Methods 291: 137-151, 2004.

19. Pavoni E, Monteriu G, Santapaola D, et al: Tumor-infiltrating B lymphocytes as an efficient source of highly specific immunoglobulins recognizing tumor cells. BMC Biotechnol 7: 70, 2007.

20. Siva AC, Kirkland RE, Lin B, et al: Selection of anti-cancer antibodies from combinatorial libraries by whole-cell panning and stringent subtraction with human blood cells. J Immunol Methods 330: 109-119, 2008.

21. Kupsch JM, Tidman NH, Kang NV, et al: Isolation of human tumor-specific antibodies by selection of an antibody phage library on melanoma cells. Clin Cancer Res 5: 925-931, 1999. 
22. Hong SY, Lee TS, Kim J, et al: Tumor targeting of humanized fragment antibody secreted from transgenic rice cell suspension culture. Plant Mol Biol 68: 413-422, 2008.

23. Thakur A and Lum LG: Cancer therapy with bispecific antibodies: clinical experience. Curr Opin Mol Ther 12: 340-349, 2010.

24. Yokota T, Milenic DE, Whitlow M and Schlom J: Rapid tumor penetration of a single-chain Fv and comparison with other immunoglobulin forms. Cancer Res 52: 3402-3408, 1992.

25. Goenaga AL, Zhou Y, Legay C, et al: Identification and characterization of tumor antigens by using antibody phage display and intrabody strategies. Mol Immunol 44: 3777-3788, 2007.
26. Douguchi J, Hashiguchi A and Sakamoto M: Construction of human monoclonal single-chain Fv antibodies against smallcell lung cancer by phage display libraries derived from cell-immunized SCID mice engrafted with human peripheral blood lymphocytes. Proteomics Clin Appl 3: 1265-1272, 2009.

27. Li ZJ, Wu WK, Ng SS, et al: A novel peptide specifically targeting the vasculature of orthotopic colorectal cancer for imaging detection and drug delivery. J Control Release 148: 292-302, 2010. 